\title{
DEDDTE

\section{1) Tecnologias computacionais para o monitoramento e a reabilitação de pessoas com doenças crônicas não transmissíveis}

Ana Carolina Bertoletti De Marchi ${ }^{1}$

\section{Resumo}

O desenvolvimento de tecnologias computacionais para o monitoramento e a reabilitação de pessoas com doenças crônicas não transmissíveis (DCNT) tem propiciado a incorporação de práticas inovadoras na saúde. O objetivo deste trabalho é apresentar duas tecnologias computacionais que visam auxiliar pacientes com DCNT: os sistemas e-Health e os exergames. Trata-se de um relato de experiência com soluções desenvolvidas pelo grupo de pesquisa Sistemas Interativos e de Informação da Universidade de Passo Fundo. Após a análise de vários estudos, os resultados indicaram que as tecnologias computacionais apresentam potencial para transforar a forma da prestação de serviços de saúde das pessoas ao longo do processo de envelhecimento.

Palavras-chave: e-Health, exergame, gerontecnologia, monitoramento, reabilitação.

\section{Introdução}

Tecnologias computacionais para o monitoramento e a reabilitação de pessoas com Doenças Crônicas Não Transmissíveis (DCNT) vêm sendo amplamente adotadas nos últimos anos, especialmente pela população idosa. $\mathrm{O}$ mundo vem vivenciando o envelhecimento populacional e iniciativas inovadoras que visem o bem-estar deste público são incentivadas.

Estas duas tendências, o envelhecimento populacional e o uso de tecnologias, fizeram surgir a gerontecnologia (BRONSWIJK et al, 2009), área que iniciou nos Estados Unidos nos anos 90 e vem conquistando cada vez mais adeptos no Brasil. A gerontecnologia estuda a interação desses dois movimentos dinâmicos na sociedade (BOUMA et al, 2007), com vistas a promover melhores condições de vida. Trata-se de um campo interdisciplinar presente em problemas simples ou complexos, direcionados à adequação dos recursos para moradia, mobilidade, comunicações, saúde, entre outros (SBtec, 2018).

Professora no Programa de Pós-Graduação em Envelhecimento Humano da Universidade de Passo Fundo. Endereço para correspondência: BR 285, São José, CEP: 99052-900, Passo Fundo, RS, Brasil. Email: carolina@upf.br 
Para a computação, o desenvolvimento de aplicações para a gerontecnologia se transformou em uma atividade desafiadora, que envolve pesquisas com diversas disciplinas como inteligência artificial, interação humano-computador, banco de dados, entre outras. Neste artigo serão abordadas duas tecnologias computacionais que visam auxiliar pacientes com DCNT: os sistemas e-Health e os exergames. O propósito é apresentar exemplos de soluções inovadoras que foram desenvolvidas para monitorar as condições de saúde e reabilitar fisicamente pessoas que necessitam de cuidados especiais.

\section{Metodologia}

Trata-se de um relato de experiência que tem como objetivo contribuir para a área de gerontecnologia, especialmente descrevendo as tecnologias computacionais desenvolvidas pelo grupo de pesquisa Sistemas Interativos e de Informação (SIN) da Universidade de Passo Fundo. Este relato é resultado da palestra ministrada no II Simpósio da Rede de Programas Interdisciplinares em Envelhecimento (REPRINTE).

\section{Sistemas e-Health}

Os sistemas e-Health (saúde eletrônica) vêm atraindo um número crescente de usuários, motivados, principalmente, pelo advento e difusão dos dispositivos móveis, bem como pelo interesse crescente na busca por uma melhor qualidade de vida, por meio de cuidados com a saúde.

A Organização Mundial da Saúde (OMS) anunciou que as soluções baseadas em e-Health "tem o potencial de transformar a forma da prestação de serviços de saúde em todo o mundo" (WHO, 2011). Os resultados da pesquisa demonstram que a maioria das regióes do mundo, incluindo países de baixa e média renda, está trabalhando ativamente em projetos e-Health ou implantou sistemas para gerenciar a adesão ao tratamento, o envio de lembretes de compromissos e/ou a realização de atividade.

Nesta perspectiva, soluções e-Health estão sendo desenvolvidas para o monitoramento de doenças crônicas não transmissíveis (DCNT), como a diabetes mellitus (DM) e a hipertensão arterial (HA). A HA é caracterizada como um fator de risco para doenças cardiovasculares (DCV), sendo uma das principais causas de morte em todo mundo. Estudos prévios vêm obtendo resultados positivos na melhora das condições de saúde de pessoas com HA, a partir de mudanças simples no estilo de vida apoiadas por soluções e-Health (TORO-RAMOS et al, 2017; ASHOORKHANI et al, 2016; ALBINI et al, 2016). As funcionalidades presentes nestas soluções visam facilitar uma ou mais rotinas do tratamento da doença. No DM algumas iniciativas já utilizam os dados armazenados para produzir relatórios capazes de detectar padrões e semelhanças em determinados comportamentos (BELLEI et al, 2018).

Diante deste contexto, foram desenvolvidas duas soluções e-Health que visam auxiliar na melhoria das condições de saúde de pessoas com HA e DM, procurando proporcionar um envelhecimento mais saudável. 
O e-Lifestyle (software com registro INPI BR51 2017 000761-6) foi concebido com o objetivo de melhorar as condições de saúde dos pacientes hipertensos (VEIGA et al, 2017) sendo uma plataforma computacional composta pelo Server e-Lifestyle e o App e-Lifestyle, que foi implementado com gamificação ${ }^{10}$. São armazenadas variáveis de peso, circunferência abdominal, altura, sono, humor, pressão arterial e prática de atividades física e realiza a avaliação de risco, a partir de uma ação conjunta que envolve o recebimento de alertas, de lembretes e o acompanhamento de um profissional da saúde. Estudos pilotos com especialistas e pacientes foram realizados para avaliar, respectivamente, as funcionalidades do e-Lifestyle (VEIGA et al, 2017) e o engajamento do paciente no uso (CECHETTI, 2018). Resultados preliminares indicaram que seria importante sua implantação no sistema público de saúde, visto que o custo com complicações em pessoas hipertensas é muito alto (VEIGA et al, 2017).

O aplicativo Soins DM (software com registro INPI BR512018001045-8) tem como objetivo acompanhar a interdependência entre fatores do tratamento de diabetes mellitus, permitindo o registro das aferições de glicemia durante todo o dia, das características e horários de ocorrência de atividades que influenciam a flutuação glicêmica (BELLEI et al, 2017). O aplicativo ainda está em fase de testes.

As soluções e-Health tem potencial para transformar a forma da prestação de serviços de saúde, a partir da avaliação de risco e do auxílio na mudança de comportamento. Contudo, o maior desafio deste mercado é manter o usuário engajado no uso a longo prazo ou de maneira recorrente, sem que haja perda de interesse.

\section{Exergames}

Exergames são jogos sérios que utilizam sensores de movimento, como o Kinect. São capazes de capturar os movimentos naturais do usuário e, assim, promover uma interação física ${ }^{12}$, a partir de um ambiente enriquecido de elementos que motivam a realização do tratamento.

Os exergames vêm sendo utilizados como uma prática inovadora na reabilitação de pessoas acometidas por doenças crônicas não transmissíveis como, por exemplo, o acidente vascular encefálico (AVE). O objetivo da reabilitação varia de jogo para jogo, podendo focar nos membros superiores (MS), membros inferiores (MI) e equilíbrio, por exemplo.

Vários estudos vêm demonstrando efeitos positivos na independência funcional dos jogadores, como no trabalho de Martel et al (2016), que também comprovou que o jogo contribuiu para o treino de atenção. O jogo utilizado neste estudo foi o Motion Rehab 2D (software com registro INPI BR512014001087-2). Trata-se do primeiro jogo da família Motion Rehab, desenvolvido pelo grupo de pesquisa SIN da UPF. Após esse, foram desenvolvidos o Motion Rehab 3D AVE (software com registro INPI BR512016001373-7) e o Motion Rehab 3D Plus.

A versão 3D AVE foi avaliada por Trombetta et al (2016), que comprovaram uma maior motivação do paciente nas sessões com o uso do jogo. Além deste estudo, Bazzanello (2017) demonstrou que a reabilitação com o exergame em pacientes pós-AVE pode ser uma alternativa eficiente para restaurar o equilíbrio e a função motora de membro superior, além de reduzir o tempo de tratamento. 
Por fim, a versão 3D Plus (TROMBETTA et al, 2018) foi desenvolvida para ser aplicada à reabilitação física de indivíduos com diferentes patologias, com um programa de atividades personalizado. O intuito é permitir a configuração do tratamento, a partir da escolha das atividades pertinentes a patologia sendo tratada. A ideia é que o jogo possibilite ao profissional configurar cada sessão de tratamento, personalizando um programa de atividades 3D específico para cada paciente. Os testes foram realizados com fisioterapeutas que concluíram que a solução customizada pode reduzir custos, ao evitar a aquisição vários jogos, e minimizar o esforço do aprendizado por parte do profissional.

Nas três versões do Motion Rehab as atividades foram pensadas a partir do tratamento convencional a fisioterapia. Para Pirovano et al (2012), o emprego do exergame, além de auxiliar no processo de reabilitação, melhora a manutenção de registros clínicos e futuros métodos de diagnóstico médico.

\title{
Conclusão
}

As tecnologias computacionais apresentam potencial para transforar a forma da prestação de serviços de saúde das pessoas ao longo do processo de envelhecimento, especialmente àquelas acometidas com DCNT. Contudo, é preciso que a experiência do usuário com estas tecnologias computacionais seja gratificante e envolvente, para garantir o engajamento ao longo de todo o tratamento.

\section{Agradecimento}

Ao Conselho Nacional de Desenvolvimento Científico e Tecnológico (CNPq).

\section{Computer technologies for the monitoring and rehabilitation of people with chronic non-communicable diseases}

\begin{abstract}
The development of computer technologies for the monitoring and rehabilitation of people with chronic noncommunicable diseases (CDNT) has led to the incorporation of innovative practices in health. The objective of this work is to present two computational technologies that aim to assist patients with CNCD: e-Health systems and exergames. This is an experience report with solutions developed by the research group Interactive Systems and Information of the University of Passo Fundo. After analyzing several studies, the results indicated that computer technologies have the potential to transform the way people provide health services throughout the aging process.
\end{abstract}

Keywords: e-Health, exergame, gerontecnology, monitoring, rehabilitation. 


\section{Referências}

ALBINI, F.; LIU, X.; TORLASCO, C.; SORANNA, D.; FAINI, A.; CIMINAGHI, R. et al. An ICT and mobile health integrated approach to optimize patients' education on hypertension and its management by physicians: The Patients Optimal Strategy of Treatment (POST) pilot study. In: 2016 38th Annual International Conference of the IEEE Engineering in Medicine and Biology Society (EMBC). IEEE; 2016. p. 517-520.

ASHOORKHANI, M.; BOZORGI, A.; MAJDZADEH, R.; HOSSEINI, H.; YOONESSI, A.; RAMEZANKHANI, A. et al. Comparing the effectiveness of the BPMAP (Blood Pressure Management Application) and usual care in self-management of primary hypertension and adherence to treatment in patients aged 30-60 years: study protocol for a randomized controlled trial. Trials. 2016 Dec 21;17(1):511.

BAZZANELLO, H.P.P. Efeitos do exergame na função motora de membro superior e no equilíbrio de pacientes pós-Acidente Vascular Encefálico. 2017;

BELLEI, E.A.; BIDUSKI, D.; CECHETTI, N.P; MARCHI, A.C.B. De. Diabetes Mellitus m-Health Applications: A Systematic Review of Features and Fundamentals. Telemed. e-Health. 2018 Feb 22;tmj.2017.0230.

BELLEI, E.A.; BIDUSKI, D.; CECHETTI, N.P.; MARCHI, A.C.B. De. Proposição de uma solução m-health para acompanhamento da interdependência entre fatores do tratamento de diabetes mellitus. In: Editora U, editor. Anais da Semana do Conhecimento UPF. Passo Fundo: 2017.

BOUMA, H.; FOZARD, J.; BOUWHUIS, D.; TAIPALE, V. Gerontechnology in perspective. Gweonthec Jounal. 2007;6(4):190-216.

BRONSWIJK, J.; BOUMA, H.; FOZARD, J.L.; KEARNS, W.D.; DAVISON G.C.; EMH, J. Defining gerontechnology for R\&D purposes. 2009;31.

CECHETTI, N.P. Uma proposição de gamificação em sistemas m-health para o engajamento dos usuários. 2018;

MARTEL, M.R.F.; COLUSSI, E.L.; MARCHI, A.C.B. De. Efeitos da intervenção com game na atenção e na independência funcional em idosos após acidente vascular encefálico. Fisioter. e Pesqui. 2016 Mar;23(1):52-58.

PIROVANO, M.; MAINETTI R, BAUD-BOVY, G.; LANZI, P.L.; BORGHESE, N.A. Self-adaptive games for rehabilitation at home. In: 2012 IEEE Conference on Computational Intelligence and Games (CIG). IEEE; 2012. p. 179-186.

SBtec. SBGtec [Internet]. 2018 [cited 2018 Sep 21];Available from: http://sbgtec.org.br/\#gerontech

TORO-RAMOS, T.; LEE, D-H; KIM, Y.; MICHAELIDES, A.; OH. T.J.; KIM, K.M. et al. Effectiveness of a Smartphone Application for the Management of Metabolic Syndrome Components Focusing on Weight Loss: A Preliminary Study. Metab. Syndr. Relat. Disord. 2017 Nov;15(9):465-473.

TROMBETTA, M.; BAZZANELLO, H.P.P.; BRUM, M.R.; COLUSSI, E.L.; MARCHI, A.C.B. De. Rieder R. Motion Rehab AVE 3D: A VR-based exergame for post-stroke rehabilitation. Comput. Methods Programs Biomed. 2017 Nov; 151:15-20.

TROMBETTA, M.; BELLEI, E.A.; RIEDER, R.; MARCHI, A.C.B. De. Motion Rehab 3D Plus: Um Exergame Customizável Aplicado à Reabilitação Física | Simpósio Brasileiro de Computação Aplicada à Saúde (SBCAS_CSBC). In: SBC, editor. Simpósio Brasileiro de Computação Aplicada à Saúde (SBCAS_CSBC). Natal: 2018.

VEIGA, J.E. Um sistema e-health para auxiliar na promoção de estilo de vida saudável em hipertensos. 2017;

WORLD HEALTH ORGANIZATION (WHO). mHealth: New horizons for health through mobile technologies: second global survey on eHealth. Switzerland: WHO Press; 2011. 